\title{
Una Perspectiva de la Integralidad. A 10 años de la creación del Consejo Social de la Universidad Nacional de La Plata
}

\section{A Perspective of Integrality. Ten years since the creation of UNLP Social Council"}

\section{Luís Adriani}

https://orcid.org/0000-0002-7541-4780

adrianiluis59@gmail.com

\section{Carlos Leavi}

https://orcid.org/0000-0002-6710-6219

carlosleavi@gmail.com

\section{Gustavo Saralegui}

https://orcid.org/0000-0001-6298-6483

gdsarale@ing.unlp.edu.ar

\author{
Inés Iglesias \\ https://orcid.org/0000-0001-9255-6265 \\ inesiglesias@gmail.com \\ Soledad Rial \\ https://orcid.org/0000-0002-3898-5023 \\ rial.soledad.sr@gmail.com \\ Verónica Veira \\ https://orcid.org/0000-0001-5236-6643 \\ veronicaveira@gmail.com
}

Consejo Social | Universidad Nacional de La Plata | Argentina

\section{RESUMEN}

Desde la recuperación democrática las Universidades Nacionales Públicas han reconocido la importancia de la Extensión como la principal expresión del Compromiso Social Universitario. En la UNLP la reforma estatutaria del 2008 implicó un importante reconocimiento a la misma al precisarse sus alcances y significado. En base a ello y en un contexto de avance y consolidación de las organizaciones sociales en la vida social y política del país, la UNLP impulsó acciones que posibilitaron una mayor inserción en los territorios y una apertura a los diferentes actores sociales mediante la conformación del Consejo Social.

A través de la construcción consensuada de una agenda comunitaria y de un permanente trabajo interactoral, el Consejo Social se propone abordar las principales problemáticas socio económicas, políticas, culturales y ambientales de la Región y acordar colectivamente estrategias de abordaje de las mismas. Desde su creación el Consejo Social ha llevado a cabo una experiencia que espera contribuir a la reorientación de las prácticas de docencia, investigación, transferencia y extensión, contribuyendo a la formación integral de los futuros profesionales, aportando a la consolidación de ciudadanías cada vez más democráticas. El propósito de este artículo es presentar la perspectiva y reflexiones surgidas a partir de la experiencia del Consejo Social, desarrollada desde su creación en 2010.

PALABRAS CLAVE Interactoralidad, Consejo Social, Integralidad 
KEY WORDS

Interactionality Social Council, Integrality

\section{ABSTRACT}

Human rights constitute the universal legal guarantee for a free and dignified life. The legislation obliges mainly States, and other agents, as the ones responsible for its realization and to act against the impediments that hinder and impede its realization. Its formal recognition is a reality that contrasts with the situations of discrimination and exclusion that are verified in various particular situations. The extension and effective realization of the right to university education does not escape the aforementioned problem. With formal recognition in international, regional and national regulations, the problem becomes evident in the various institutions of university education.

The article aims to reflect on some guidelines of the critical theory in order to guarantee the right to university education. It focuses on the analysis of the "institutional habitus" as one of the key factors for university level institutions to exercise their responsibility as a key agent in their guarantee. Finally, it refers to the institutional project of preventive nature of academic failure developed at the National University of Cuyo (UNCuyo) called Student Academic Career (TRACES). 


\section{PRESENTACIÓN}

La presente contribución tiene como objetivo describir la experiencia desarrollada por el Consejo Social de la Universidad Nacional de La Plata UNLP desde su creación en 2010, exponer los principales conceptos en los que fundamenta su perspectiva y práctica de la extensión y los interrogantes que han surgido de problematizar el conjunto de las funciones universitarias a partir de la misma.

Para situar las experiencias y acciones del Consejo Social a lo largo de estos 10 años, resulta necesario recorrer el proceso de recuperación y reflexión en torno de la Extensión Universitaria, a lo largo de las últimas décadas y también situar históricamente en nuestra región en particular y en el país en general las propuestas, miradas y luchas en torno a lo que hoy concebimos como integralidad universitaria y su correspondiente construcción de ciudadanías democráticas dentro y fuera de los claustros universitarios.

La UNLP fue fundada por y sobre el ideario educativo universitario de Joaquín V. González, y estableció ya en 1905 la necesidad de vincular la vida académica con la realidad social que la contiene y que le da sentido. Esa impronta quedó establecida en el Estatuto fundacional que define, por primera vez en el país, "a la extensión del conocimiento, la acción y los servicios hacia los sectores populares como una de las misiones fundamentales de esa universidad" (Gezmet, Compendio bibliográfico 2015, pág. 6)

En la misma línea, la resolución de la 7ma. Asamblea Universitaria de 1913, expresa que "toda persona en la república, cualquiera sea su desarrollo intelectual, debe hallar una puerta abierta para ingresar en la universidad, en procura de algún saber, o de ampliación o intensificación del saber adquirido" (Picco, 2007, pág. 9)

\section{UN SIGLO DE LUCHAS POR LA DEMOCRATIZACIÓN UNIVERSITARIA}

Estas miradas sobre la misión social y la democratización del saber, el acceso y los vínculos comunitarios en las Universidades se profundizó a partir de la Reforma de 1918, y se extendió desde Córdoba a todas las Universidades Argentinas y a América Latina. 
Vale recordar que su documento más importante, el "Manifiesto Liminar" se dirige a los "hombres libres de Sud América", afirmando en ese momento como tema central que "nuestro régimen universitario -aun el más reciente- es anacrónico. Está fundado sobre una especie de derecho divino;" (...) por esto lo que se "reclama es un gobierno estrictamente democrático..." (Manifiesto Liminar de la Reforma Universitaria, 1918). Con esta cita buscamos exponer que las reflexiones de este artículo son parte de una tradición de luchas y disputas por el sentido de la universidad que viene de larga data y continúa en la actualidad.

\section{Estas miradas sobre la misión social y la democratización del saber, el acceso y los vínculos comunitarios en las Universida- des se profundizó a partir de la Reforma de 1918, y se exten- dió desde Córdoba a todas las Universidades Argentinas y a América Latina.}

Durante todo el siglo XX en diferentes contextos sociopolíticos continuaron estas tensiones y debates en torno al rol universitario, incluso cuando el Estado definió su gratuidad hace 71 años, en el primer gobierno de Juan Domingo Perón. Fueron muchos y muy diversos los procesos de las Universidades a lo largo de ese siglo, y nos interesa recuperar los debates y lineamientos a los que las Universidades Latinoamericanas arribaban en los años '70, de la mano de referentes como Paulo Freire, Darcy Ribeiro y tantos otros.

Como cita Cano Menoni, las Universidades reunidas en la "II Conferencia Latinoamericana de Difusión Cultural y Extensión Universitaria" en 1972, concluían: “La extensión universitaria deberá: mantenerse solidariamente ligada a todo proceso que se de en la sociedad tendiente a abolir la dominación interna y externa, y la marginación y explotación de los sectores populares de las sociedades. Estar despojada de todo carácter paternalista y meramente asistencialista, y en ningún momento ser transmisora de los patrones culturales de los grupos dominantes. Ser planificada, dinámica, sistemática, interdisciplinaria, permanente, obligatoria y coordinada con otros factores sociales que coincidan con sus objetivos, y no sólo nacional, sino promover la integración en el ámbito latinoamericano". (Cano Menoni, 2014, pág. 3)

Vemos entonces, en esta definición, un claro sentido transformador asignado a la Extensión Universitaria, una mirada crítica sobre la tendencia de poner el conocimiento académico por encima de otros saberes y la voluntad de formalizar los procesos de la vinculación comunitaria, para convertirlos en instrumentos de la transformación.

A modo de síntesis, traemos la definición que presenta el profesor chileno Domingo Piga, en su ponencia en dicha Conferencia: 
(La Extensión es) "aquella que interrelaciona activa y creadoramente la universidad con la comunidad nacional, aquella que contribuye por medio de la cultura universitaria (ciencia, arte, técnica) a transformar el mundo para crear otro mundo cuyos sistemas y estructuras socioeconómicas sean más justos, más dignos, y más ético". (Tunnermann Berheim, 1978, pág. 109)

En este sentido, es válido destacar que en la UNLP se dio un proceso entre mayo de 1973 y octubre de 1974 de participación de los trabajadores nodocentes en la gestión de la propia Universidad, que terminó abruptamente con el asesinato del Director del Departamento Central de Planificación, Carlos Miguel, y el Secretario de Supervisión Administrativa, Rodolfo Achem. Estos compañeros habían sido claros referentes e impulsores de las discusiones en torno a forjar nuevos proyectos universitarios que, en este caso, se expresaron con claridad en el documento "Bases para la nueva universidad" producido por la Federación Universitaria de la Revolución Nacional, aprobado por una ordenanza que entre sus considerandos exponía como central entre otras cosas, que "era necesario superar una etapa signada por una enseñanza estanca, meramente acumulativa de conocimientos y al servicio de un individualismo utilitario". (Ponte)

Las dictaduras militares a lo largo de toda Latinoamérica interrumpieron violenta y deliberadamente estos procesos. Durante todos los gobiernos dictatoriales se sucedieron los ataques para destruir los proyectos de desarrollo y vinculación de las Universidades; donde baste mencionar, solo a modo ilustrativo, la llamada "Noche de los bastones largos", con el dictador Juan Carlos Onganía como presidente de facto; pero la represión durante la última dictadura cívico militar fue, sin dudas, la más sangrienta, extendida y coordinada, y durante ese período las Universidades Públicas y sus comunidades fueron objetivos claros. La UNLP es la mayor Universidad con estudiantes, nodocentes y docentes desaparecidos de todo el país.

La recuperación democrática a partir de diciembre de 1983 implicó un esfuerzo significativo para las Universidades Públicas Nacionales, recuperación que en la UNLP implicó la reapertura de carreras, el desmantelamiento de las condiciones restrictivas de ingreso y la recomposición de la pluralidad en la vida política y académica de sus claustros.

\section{La recuperación democrática a partir de diciembre de 1983 implicó un esfuerzo significativo para las Universidades Pú- blicas Nacionales, recuperación que en la UNLP implicó la re- apertura de carreras, el desmantelamiento de las condiciones restrictivas de ingreso y la recomposición de la pluralidad en la vida política y académica de sus claustros.}


En relación a la Extensión Universitaria, en nuestra Universidad la Secretaria de Extensión devino durante la Dictadura en Secretaría de Extensión Cultural, hasta 1991 en que retomó su denominación anterior, pero mantuvo el sesgo de "Extensión Cultural", aunque comenzaron a recuperarse algunas experiencias que sostenían a la Extensión como se concebía antes del Golpe de Estado. (Picco, 2007)

\section{LA DÉCADA DEL 90 Y LAS POLÍTICAS NEOLIBERALES: DE LA EX- TENSIÓN CULTURAL A LOS SERVICIOS A TERCEROS.}

En los convulsionados años 90 y ante un desarrollo regional de políticas de corte neoliberal, que llegaron incluso a intentar arancelar la educación universitaria en varios momentos, diferentes políticas fueron empujando a las Universidades a buscar recursos propios para sostener sus actividades, incluso para el pago de servicios básicos.

En ese marco comienzan a crecer procesos de transferencia a empresas, servicios a terceros y una oferta de posgrados arancelados, tendientes a sostener el funcionamiento frente al ahogo presupuestario y las políticas que beneficiaban esas iniciativas. Frente a esto, diversos sectores de la comunidad universitaria, con especial protagonismo del movimiento estudiantil, resisten el ajuste, cuestionan estas medidas y plantean que la Universidad Pública debe hacer frente a estas políticas fortaleciendo sus vínculos comunitarios, y que la Extensión Universitaria es la principal herramienta para eso.

Estas circunstancias encontraron una bisagra histórica en la crisis social, económica y política que se terminó de desencadenar a finales de 2001 y que se extendió fuertemente durante 2002 en Argentina, lo cual generó las condiciones del proceso actual en la construcción de las agendas extensionistas. Es importante reconocer este punto como el inicio de un proceso de "reaprendizaje", un "empezar de cero", para la comunidad universitaria, de muchos procesos y prácticas que habían sido cancelados por la Dictadura.

\section{Estas circunstancias encontraron una bisagra histórica en la crisis social, económica y política que se terminó de desenca- denar a finales de 2001 y que se extendió fuertemente duran- te 2002 en Argentina, lo cual generó las condiciones del pro- ceso actual en la construcción de las agendas extensionistas.}

Docentes, nodocentes, graduadxs y estudiantes se volcaron en esos años a la construcción de proyectos para aportar respuestas desde los diferentes campos del conocimiento, al abordaje de problemas de los sectores más castigados por la crisis, asistiendo a comedores, centros de salud, instituciones barriales, escuelas, entre muchos otros. Esta primera 
elaboración podría caracterizarse como "Extensión Solidaria”, ya que la voluntad de colaborar fue su principal motor, y la formación para esas prácticas fue surgiendo en la medida en que la realidad la demandaba.

La realidad de cada territorio, de cada problemática que se intentaba abordar, fue poniendo en evidencia la necesidad de la interdisciplina. Quedaba claro que cada disciplina por separado era incapaz de abordar la complejidad de los problemas de la comunidad, y eso llevó a los equipos a buscar, primero artesanalmente, y luego a partir de políticas institucionales, la vinculación con otros grupos, otras disciplinas.

En el mismo sentido, con el paso del tiempo y la elaboración sobre la práctica, se recuperó el concepto de la Extensión como función sustantiva, como compromiso y responsabilidad de la Universidad y no como un hecho voluntario y separado del resto del quehacer universitario. A partir de allí, se avanzó en profundizar qué significaba la Extensión como función; se estableció la necesidad de vincularla a la producción de conocimiento de cada área y disciplina y también se trabajó fuertemente en diferenciarla de la transferencia y servicios a terceros, definiendo sus objetivos y principales destinatarios.

\section{En el mismo sentido, con el paso del tiempo y la elaboración sobre la práctica, se recuperó el concepto de la Extensión como función sustantiva, como compromiso y responsabili- dad de la Universidad y no como un hecho voluntario y sepa- rado del resto del quehacer universitario.}

Un paso siguiente, aún en proceso pero con importantes avances, fue reconocer que la Universidad no es portadora de un saber más relevante o significativo que otros, al momento de abordar temáticas vinculadas al desarrollo y la garantía de derechos; y eso puso en evidencia la necesidad de interactoralidad, la problematización colectiva y la construcción de proyectos conjuntamente con los actores de las comunidades, sumando a la Universidad como un actor más, aprendiendo a escuchar y favoreciendo la construcción de consensos.

La institucionalización de estos debates alcanzó su maduración en la reforma estatutaria de la UNLP de $2008^{1}$, en cuyo Preámbulo se establece:

"La tercera (la Extensión), debatida y consensuada con el conjunto de la comunidad, perseguirá contribuir a la búsqueda de respuestas a problemas sociales, fundamentalmente de aquellos sectores más vulnerables por no tener sus derechos esenciales garantizados. La Extensión Universitaria será el principal medio de la Universidad Nacional de La Plata para lograr su función social, contribuyendo al tratamiento de los problemas que afectan al bienestar de la comunidad, la recons- 
trucción del tejido social, el desarrollo económico sustentable y el fortalecimiento de la identidad cultural."

Mientras en el Capítulo III, en su artículo 17, establece con claridad "la Universidad reconoce como una de sus funciones primordiales la extensión universitaria, entendida como un proceso educativo no formal de doble vía, planificada de acuerdo a intereses y necesidades de la sociedad, cuyos propósitos deben contribuir a la solución de las más diversas problemáticas sociales, la toma de decisiones y la formación de opinión, con el objeto de generar conocimiento a través de un proceso de integración con el medio y contribuir al desarrollo social".

\section{EL CONSEJO SOCIAL COMO EXPERIENCIA DE LOS NUEVOS (VIEJOS)} PARADIGMAS DEL MODELO UNIVERSITARIO.

Como venimos exponiendo en las referencias históricas de los movimientos universitarios y sus modelos de desarrollo en disputa, el Consejo Social podría pensarse como parte de esas trayectorias ${ }^{2}$, de esas historias de luchas múltiples por un modelo de universidad en vínculo permanente con las necesidades y problemáticas comunitarias.

El Consejo Social de nuestra Universidad nació como asesor del Presidente, convocando a organismos públicos, actores guberna mentales, sindicatos y movimientos sociales, sumando también a representantes de facultades y colegios. Desde el comienzo se pensó como un espacio de articulación concreta entre la Universidad y la Comunidad, para dar respuestas a las principales problemáticas sociales de la región, que se definió contemplando los municipios de La Plata, Berisso, Ensenada, Magdalena, Brandsen y Punta Indio.

Los fines del Consejo Social establecidos fueron: reunir a todos los actores de la región para analizar las principales problemáticas socio económicas, políticas, culturales, y ambientales, y discutir conjuntamente posibles estrategias de abordaje mediante políticas locales y nacionales; al mismo tiempo se esperaba que este vínculo y el desarrollo de los proyectos aportar a reorientar las propuestas de docencia, investigación y extensión de los equipos.

El Consejo Social se estructura a partir de una Dirección General, Direcciones específicas, un espacio Plenario, donde sus integrantes aportan su mirada y experiencia en la totalidad de los temas de agenda, y en Comisiones de Trabajo generadas a partir de los temas de agenda. Integran el Consejo Social Dependencias del Gobierno de la Provincia de Buenos Aires, representantes de las cámaras legislativas, los municipios de la Región Capital, centrales sindicales, organizaciones territoriales, cooperativas, organismos de ciencia y tecnología y diferentes actores de la comunidad universitaria.

La agenda de trabajo del Consejo ha ido creciendo a lo largo de los años, y actualmente cuenta con seis Comisiones permanentes que

\footnotetext{
${ }^{1}$ Estatuto de la UNLP. Disponible en https://unlp.edu.ar/gobierno/estatuto_unlp-4287

2 Aquí se produce un diálogo con el nombre de esta revista, una articulación que no es casual porque la experiencia en el dictado del Seminario en la Especialización Docente ha sido un paso fundamental en la problematización de los alcances del Consejo Social dentro de la propia universidad.
} 
abordan temas diversos como: Tierra, Vivienda y Hábitat, Economía Popular, Social y Solidaria, Salud, Niñez, Adolescencia y Juventud, Educación y Derechos Migrantes. Otros temas, priorizados en diferentes contextos han dado origen a otras Comisiones, que funcionaron durante el desarrollo de los mismos, y también a convocatorias a proyectos, gestionadas por el propio Consejo Social.

Los grandes ejes de su desarrollo pueden agruparse en Diplomaturas Universitarias, Promoción de Derechos, Fortalecimiento de la Economía Popular, Social y Solidaria y Promoción de la gestión participativa de Políticas Públicas y Conflictos ${ }^{3}$.

Con 10 años de pleno crecimiento y funcionamiento, el Consejo Social es un lugar donde las más diversas entidades son convocadas a discutir sus problemáticas dentro de la Universidad, es decir un lugar de articulación del Estado con amplios sectores del tejido social. Se postula como una herramienta, articuladora, propositiva y eficaz que está preparada, entre otras cosas, para hacer frente a situaciones de emergencia social, como las inundaciones del 2013 y actualmente, en el año 2020, la pandemia del COVID 19 y las disposiciones de ASPO y DISPO.

\section{Con 10 años de pleno crecimiento y funcionamiento, el Con- sejo Social es un lugar donde las más diversas entidades son convocadas a discutir sus problemáticas dentro de la Univer- sidad, es decir un lugar de articulación del Estado con amplios sectores del tejido social.}

Esto se ha logrado, y se logra, nutriendo el pensamiento crítico, la autonomía institucional y la capacidad académica desarrollada por la Universidad en cada una de sus facultades y dependencias, con el aporte de funcionarios públicos, trabajadores ocupados y desocupados, organismos de ciencia y tecnología, e integrantes de la comunidad toda.

\section{LA PERSPECTIVA DE EXTENSIÓN UNIVERSITARIA DEL CONSEJO} SOCIAL: NUESTRA EXPERIENCIA EN EL CAMINO HACIA LA ARTICULACIÓN INTEGRAL

Desde nuestra perspectiva, la extensión universitaria ha condensado y expresado los diferentes modos en que la universidad organiza, en diferentes coyunturas histórico-sociales, su compromiso con los procesos sociales, culturales, económicos y políticos de su contexto (Cano Menoni, 2014, pág. 4). Por eso afirmamos que sigue siendo la principal herramienta de formación para la integralidad, y la puerta para re pensarnos en relación a la comunidad.

De lo descrito en los apartados anteriores interesa en esta oportu-

${ }^{3}$ https://unlp.edu.ar/consejo_social 
nidad detenernos en cuatro de los conceptos con los que coincidimos para el desarrollo de la extensión universitaria en su relación con las diferentes funciones de la Universidad: agenda comunitaria, interactoralidad, integralidad y democratización de la educación universitaria.

Concebimos la interactoralidad en los términos del nuevo Estatuto de la UNLP, que consolida un proceso de reflexión que asume el DIÁLOGO DE SABERES (doble vía) que implica necesariamente el reconocimiento de los saberes, recorridos y sentidos de la totalidad de los actores que componen la comunidad, y que desarrollan propuestas y proyectos, muchas veces sin vinculación con la Universidad. El reconocimiento efectivo de la necesidad de sumar a esos procesos, con humildad, con escucha; asumiendo que sentar a la comunidad a la mesa, a planificar y cogestionar, implica un compromiso permanente $y$, hasta por momentos, incómodo para las lógicas universitarias, es el sentido del Consejo Social, y es lo que lo ha convertido en una herramienta reconocida por muy diversos sectores de la comunidad. Aquí es importante reconocer que estos avances sólo han sido posibles a partir de la decisión política institucional de la Presidencia de la UNLP a lo largo de los últimos 10 años, que ha tomado la decisión consciente y deliberada de poner la voz y el prestigio de nuestra Universidad al servicio de los más vulnerables, asumiendo muchas veces cuestionamientos de los sectores más favorecidos por las políticas que profundizan la desigualdad.

\section{Concebimos la interactoralidad en los términos del nuevo Estatuto de la UNLP, que consolida un proceso de reflexión que asume el DIÁOGO DE SABERES (doble vía) que implica necesariamente el reconocimiento de los saberes, recorridos y sentidos de la totalidad de los actores que componen la co- munidad, y que desarrollan propuestas y proyectos, muchas veces sin vinculación con la Universidad.}

En el mismo sentido, entendemos por agenda comunitaria la construcción consensuada y sistematizada de los ejes prioritarios que responden a demandas de los diferentes actores que integran el Consejo sobre los cuales trabajar en un período determinado. Como planteamos, la irrupción del actor social y comunitario, y del contexto social, en la construcción de la agenda universitaria cuestionan la concepción de liderazgo estereotipado y las prácticas establecidas, fuertemente condicionadas por los requisitos de las agencias de investigación, las disposiciones inherentes a los cargos y las limitaciones de las dedicaciones docentes y de investigación.

En el Consejo Social la interactoralidad y la agenda comunitaria son constitutivas de su práctica. La definición de la agenda en el ple- 
nario y el trabajo en las comisiones implican un proceso de construcción conjunta de acuerdos y de acciones en las que convergen los distintos actores con sus diferentes saberes: integrantes de organizaciones sociales, extensionistas, investigadores, docentes, graduadxs, estudiantes, nodocentes, representantes de instituciones públicas y de órganos de gobierno.

En este camino de reaprender la interdisciplina y la interactoralidad, avanzamos en la necesidad de la integralidad como concepción y práctica que incorpora los aprendizajes y perspectivas que surgen del compromiso con la agenda comunitaria a las prácticas de enseñanza, investigación, transferencia e innovación, superando la fragmentación y promoviendo una transformación institucional que no asigna un "casillero" a su función social, sino que la desarrolla en todos y cada uno de sus territorios.

En este camino de reaprender la interdisciplina y la interactoralidad, avanzamos en la necesidad de la integralidad como concepción y práctica que incorpora los aprendizajes y perspectivas que surgen del compromiso con la agenda comunitaria a las prácticas de enseñanza, investigación, transferencia e innovación, superando la fragmentación y promoviendo una transformación institucional que no asigna un "casillero" a su función social, sino que la desarrolla en todos y cada uno de sus territorios.

La agenda comunitaria exige, como mínimo, procedimientos interdisciplinarios, para lo cual se requiere no solo de saberes, sino también de una formación a tal fin, de una organización institucional y de una disponibilidad de recursos acorde. En este sentido el camino hacia la verdadera interdisciplinariedad y la integralidad implica desandar el camino de la reproducción de la fragmentación y la atomización de saberes y prácticas de investigación, de enseñanza y de gestión universitarias.

Esto ha sido, y sigue siendo, ampliamente elaborado por diversas corrientes en Latinoamérica en los últimos años, siendo quizás la mayor referencia la experiencia en la Universidad de la República en Uruguay, en donde, durante la gestión de Rodrigo Arocena como Rector, entre 2006 y 2014, se avanzó en el planteo de una Segunda Reforma Universitaria con eje en la integralidad de las prácticas universitarias, orientadas por su compromiso social expresado a través de la Extensión crítica y transformadora.

Coincidimos con esa mirada, y entendemos que nos encontramos ante la posibilidad de recuperar experiencias y aprendizajes de nuestro Consejo Social y otros espacios en los que se produce conocimiento con 
otrxs, como los Centros Comunitarios de Extensión, los proyectos con amplia trayectoria y vínculos consolidados, asumiendo el desafío de la integralidad, entendida como "...la articulación de actores sociales y universitarios. Por un lado, con la construcción y abordaje de los sujetos y objetos de estudio con miradas interdisciplinarias y por otro, con la posibilidad de construcción intersectorial, interinstitucional de propuestas que resuelvan problemáticas concretas". (Arocena, y otros, pág. 26)

Por último, otro elemento de la construcción del Consejo Social que nos parece importante recuperar es la democratización. Tanto en lo que hace a los espacios de debate, como al desarrollo de los proyectos y de las políticas públicas con las que se ha vinculado a lo largo de los años, el trabajo del Consejo se basa en profundizar las prácticas de la democracia participativa, generando condiciones para construcción de consensos y aportando capacidades para ello a todxs Ixs involucrados. Por ello, a nuestro entender, recuperar estos aprendizajes, aportarlos a repensar las prácticas universitarias, también nos involucra en la construcción de ciudadanías cada vez más democráticas.

\section{el trabajo del Consejo se basa en profundizar las prácticas de la democracia participativa, generando condiciones para construcción de consensos y aportando capacidades para ello a todxs Ixs involucrados.}

Aquí resulta muy interesante volver a la ponencia de Domingo Piga, de la II Conferencia Latinoamericana de Extensión Universitaria y Difusión Cultural, y recuperar el aporte de Domingo Piga, cuando describe lo que llama la "Extensión Intrauniversitaria" y sostiene que la misma se propone ampliar la formación humanista de los miembros de la comunidad universitaria para que estos sean realmente capaces de tener esa visión totalizadora del hombre en la sociedad. “De este modo, añade Piga, se activa la intercomunicación de los universitarios, estos valorarán las otras disciplinas universitarias, trabajarán en equipo y multidisciplinariamente, y llegarán a ser realmente capaces de un análisis crítico e integral para servir mejor a la sociedad". (Tunnermann Berheim, 1978, pág. 109)

\section{A MODO DE CIERRE Y DE APERTURA AL DEBATE ${ }^{4}$}

El Consejo Social enfrenta distintos desafíos, tanto externos como internos, que podríamos sintetizar en los siguientes ejes: repensar cómo construimos nuestros trayectos educativos, con y para quiénes; cómo se vincula el sistema de Ciencia y Tecnología con la agenda comunitaria; cuál es el espacio para la Tecnología de Apropiación Social y, fundamen-

\footnotetext{
${ }^{4}$ El presente artículo busca enunciar algunos de los interrogantes que dan cuenta de la problematización que surge a partir de las múltiples experiencias del Consejo Social, somos conscientes que se trata de la apertura de un conjunto de debates que no se agotan en estas líneas, ya que su complejidad y profundización está en proceso de desarrollo.
} 
talmente, nos lleva a preguntarnos qué profesionales y qué ciudadanos estamos formando cuando no avanzamos sobre esos debates.

Estos desafíos nos posicionan al mismo tiempo ante la exigencia de una problematización continua, reflexiva y crítica, como así también ante la necesidad de emprender caminos creativos y propositivos que conllevan la construcción de nuevas herramientas.

La gestión del Consejo Social adquiere un rol central en la articulación de necesidades (de intervención, de investigación, de participación de cátedras) con los diferentes actores, sectores y territorios. Desarrolla un recorrido que hasta el momento resulta muy valioso, y sus aprendizajes muy significativos; sin embargo, se mantiene en "Ios márgenes" de la propia universidad y sus prioridades centrales.

Entonces nos encontramos ante el desafío de ampliar el alcance del Consejo Social, no sólo en relación a los proyectos que desarrolla, sino a la interpelación de la propia Universidad, sus claustros, facultades y dependencias. Como plantea Judith Sutz, de alguna manera estamos construyendo un "espacio de preguntas recíprocas" que nos permitan ir de las acciones a la planificación de políticas, de las prácticas a las teorías, produciendo conocimiento con los/as referentes barriales y/o en políticas públicas para transformar la realidad y la Universidad. (Arocena, y otros, pág. 52) 
Arocena, R., Tommasino, H., Rodríguez, N., Sutz, J., Álvarez Pedrosian, E., \& Romano, A. (s.f.). Cuaderno $N^{\circ}$ 1. Integralidad: tensiones y perspectivas. Montevideo, Uruguay.

Cano Menoni, J. A. (2014). "La Extensión Universitaria en la transformación de la universidad latinoamericana del siglo XXI: disputas y desafíos". Obtenido de http://biblioteca.clacso.edu.ar/clacso/becas/20141202093928/ensayo_cano_ premio_pedro_krotsch.pdf

Gezmet, S. (Compendio bibliográfico 2015). “Evolución histórica-crítica de la Extensión Universitaria. Proceso de institucionalización de la Extensión de la UNC en los distintos momentos históricos. Asignatura Extensión Universitaria. Universidad Nacional de Córdoba". Obtenido de https://ffyh.unc.edu. ar/extension/wp-content/uploads/sites/2/2018/08/Compendio-Bibliografico-2015-asignatura-extension-universitaria.pdf

Manifiesto Liminar de la Reforma Universitaria. (21 de junio de 1918). Obtenido de http://www.fi.uba.ar/sites/default/files/Manifiesto\%20Reforma\%20 Universitaria-.pdf

Picco, S. (2007). “Extensión y formación docente en los orígenes de la Universidad Nacional de La Plata (1906-1920)". Question, Vol. 1, N 13. En Memoria Académica. Obtenido de http://www.memoria.fahce.unlp.edu.ar/art_revistas/ pr.8521/pr.8521.pdf

Ponte, I. (s.f.). "La participación de los trabajadores en la conducción de la Universidad". En Cultura y Política La Yoli de Lanús La Revista del Gremio Nodocente de la UNLa No 8. Asociación de Trabajadores y Trabajadoras de la Universidad Nacional de Lanús. Obtenido de http://www.atunla.org/la-yoli-de-lanus/ano2/n-8/la-participacion-de-los-trabajadores-en-la-conduccion-de-la-universidad

Tunnermann Berheim, C. (1978). “El nuevo concepto de la Extensión Universitaria y difusión cultural y su relación con las políticas de desarrollo cultural en América Latina". En Anuario de Estudios Centroamericanos Nº 4 Pág. 93-126. 